\title{
Osteoporotic Characteristics Persist in the Spine of Ovariectomized Sheep after Withdrawal of Corticosteroid Administration
}

\author{
Mohammad-Reza Zarrinkalam, ${ }^{1,2}$ Christopher G. Schultz, ${ }^{3}$ \\ Ian H. Parkinson, ${ }^{2,4,5}$ and Robert J. Moore ${ }^{1,2,5}$ \\ ${ }^{1}$ The Adelaide Centre for Spinal Research, SA Pathology, Adelaide, Australia \\ ${ }^{2}$ Hanson Institute, SA Pathology, Adelaide, Australia \\ ${ }^{3}$ Bone Densitometry Unit, Department of Nuclear Medicine, Royal Adelaide Hospital, Adelaide, Australia \\ ${ }^{4}$ Bone and Joint Research Laboratory, SA Pathology, Adelaide, Australia \\ ${ }^{5}$ Discipline of Anatomy and Pathology, University of Adelaide, Adelaide, Australia
}

Correspondence should be addressed to Mohammad-Reza Zarrinkalam, reza.zarrinkalam@health.sa.gov.au

Received 18 July 2012; Accepted 31 August 2012

Academic Editor: Jun Iwamoto

Copyright (C) 2012 Mohammad-Reza Zarrinkalam et al. This is an open access article distributed under the Creative Commons Attribution License, which permits unrestricted use, distribution, and reproduction in any medium, provided the original work is properly cited.

\begin{abstract}
A validated ovine model of osteoporosis achieves severe bone loss in a relatively short period. This study investigated if osteoporotic features persist in this model after cessation of corticosteroid administration. Methods. Osteoporosis was induced in nine ewes by chronic corticosteroid injection, ovariectomy, and low calcium diet. Six ewes were used as controls. Bone mineral density (BMD) of the lumbar spine (LS) and body weight were assessed at regular intervals. After five months, corticosteroid treatment was withdrawn systematically over one month. Three months later, all animals were euthanised, and the LS was collected for histomorphometric analysis. Results. BMD in the LS of osteoporotic sheep was $25 \%$ lower than control sheep. Body weight of osteoporotic sheep was reduced in the first month of the corticosteroid withdrawal period but returned to baseline level thereafter. Trabecular bone volume of LS in osteoporotic sheep was $27 \%$ lower than controls and showed a heterogeneous structure. Conclusions. Osteoporotic characteristics remain in the vertebra after ceasing corticosteroid administration providing an opportunity to evaluate potential systemic or local treatments in vivo under realistic physiological conditions. The microstructural arrangement of vertebral trabecular bone in sheep is similar to humans demonstrating further relevance of this model for preclinical investigations.
\end{abstract}

\section{Introduction}

Osteoporosis is a generalised condition of decreased bone mass that is characterised by a significant increase in the likelihood of skeletal fractures $[1,2]$. Of all the fractures associated with osteoporosis, just under $50 \%$ occur in the spine with the remainder fairly evenly distributed between the hips, wrists, and other skeletal sites [3]. According to the World Health Organisation, osteoporosis is a "silent epidemic" [4] which is increasing as the population ages. It is estimated that 10 million people in the USA and 1.9 million people in Australia already have osteoporosis, and it is expected that these numbers will double over the next decade [5].

Various animal models have been utilised to investigate the osteoporotic condition in order to facilitate preclinical testing of pharmaceutical agents and to evaluate other treatment options. The ovariectomised rat is recognised by the U.S. Food and Drug Administration as a suitable animal model [6] but due to limitations, such as lack of true lamellar bone and Haversian systems and being relatively small, there is still a need for a complementary large animal model to improve orthopaedic procedures by developing new implants and fixation strategies [7]. In 
our experience, osteoporosis can be induced in sheep with ovariectomy, low calcium diet, and weekly corticosteroid injection for up to 6 months [8] and although this model (as is the case with all animal models) does not specifically mimic either postmenopausal or glucocorticoid-induced osteoporosis in humans, it does achieve severe bone loss in a relatively short period and displays many pathophysiological features that are similar to the human osteoporotic state [8-10]. Further, it offers the convenience of conducting spinal surgery and preclinical testing of devices (including pharmaceuticals at similar doses) intended for use in humans under highly relevant clinical conditions. It is our view that this model would be further enhanced by terminating steroid administration after induction of osteoporosis to remove potential confounding interactions of the corticosteroids with proposed interventions. Thus, the aim of this study was to characterise the morphological features of vertebral bone in this model after withdrawal of the corticosteroid injection regimen $[11,12]$. The hypothesis tested was that vertebral BMD and bone architecture, as indicators of bone quality [13-15] in osteoporotic sheep, remain unchanged after cessation of corticosteroid administration.

\section{Materials and Methods}

2.1. Animal Preparation. With Institutional Animal Ethics Committee approval nine 3-year-old Merino ewes, (mean weight $66.3 \pm 8 \mathrm{~kg}$ ) were bilaterally ovariectomised under general anaesthesia. The animals were housed in barren pens and fed a low calcium diet with weekly intramuscular injections of corticosteroid [8]. These sheep comprised the osteoporotic group. Bone mineral density (BMD) of the lumbar spine (L2 to L5) was assessed monthly by DXA and when it was reduced by at least $25 \%$ from baseline, the corticosteroid was withdrawn to permit resumption of natural cortisol production. The animals were maintained on a low calcium diet. Since rapid withdrawal of corticosteroid can result in acute adrenal insufficiency [16], it was systematically reduced by $50 \%$ each week until the end of the fourth week when it was stopped altogether. Six age-matched control sheep (mean weight $65.7 \pm 7 \mathrm{~kg}$ ) underwent sham abdominal surgery and were fed a normal diet. All animals were observed for their appetite and general wellbeing during the experiment and weighed weekly, and the lumbar spine BMD was assessed monthly by DXA. Three-months after withdrawal of corticosteroid in the osteoporotic group, all animals were humanely killed and their lumbar spines collected. Thus this interval includes the healing and regeneration phase which is critical following any orthopaedic intervention, including fixation of implants.

2.2. Bone Mineral Density (BMD). Dual-energy X-ray absorptiometry (DXA) was conducted on the lumbar spine (L2 to L5) of all animals using a Hologic QDR 2000 scanner (Waltham, MA, USA. s/n 2263 software version $7.10 \mathrm{~b}$ with standard array mode acquisition $-1.8 \mathrm{uSv}$ effective $\mathrm{X}$ ray radiation dose) at the selected time points. Animals were positioned supine on the scanner table under general anaesthesia or immediately after death. The upper limbs were folded and secured to the chest with masking tape, and the lower limbs were extended and held down loosely with the tape.

2.3. Micro CT. The fourth lumbar vertebra of each animal was cleaned of soft tissue and the spinous processes removed using a band saw. The vertebrae were fixed in 10\% formalin for one week, transferred to $70 \%$ ethanol, and wrapped in polythene film to minimise dehydration during micro-CT imaging (SkyScan 1076 in vivo scanner, SkyScan, Kontich, Belgium). Projection images were obtained at $100 \mathrm{kV}$ and $75 \mu \mathrm{A}$ with a $1 \mathrm{~mm}$ aluminium filter and a pixel size of $17.4 \mu \mathrm{m}$ in all three spatial planes. A rotation step of $0.5^{\circ}$ was used between each image. From this series of projection images, two-dimensional tomographs were reconstructed, orthogonal to the imaging plane, with NRecon software (version 1.5.1.3, Skyscan, Kontich, Belgium). On average, there were 2020 tomographs for each vertebra.

For histomorphometric analysis, the volume of interest (VOI) was defined for each vertebral body. The first and last 200 tomographs from each vertebra were excluded to ensure the endplate was not included in the analysis. The remaining tomographs were divided into three equal zones comprising two subendplate zones and one central zone similar to human studies $[11,12]$. Each zone was further divided into the left and right halves (Figure 1). Each VOI was saved as a new smaller data set to aid data processing. Using CTAn software (version 1.8.1.2, Skyscan, Kontich, Belgium), the following morphometric descriptors of trabecular bone were measured from the VOI of each zone: tissue volume (TV), bone volume (BV), percent bone volume (BV/TV), tissue surface (TS), bone surface (BS), intersection surface (i.S), bone surface/volume (BS/BV), bone surface density (BS/TV), trabecular thickness (Tb.Th), trabecular separation (Tb.Sp), and trabecular number (Tb.N) [17].

Differences between vertebral regions, body weight, $\mathrm{BMD}$, and bone morphometric parameters in control and osteoporotic sheep were tested using one-way analysis of variance with Tukey's post hoc comparison of means (SAS, SAS Institute, Cary, NC). Statistical significance was set at $P<0.05$.

\section{Results}

3.1. General Observations. The weight of animals during the induction of osteoporosis did not change significantly (Figure 2) but with the gradual withdrawal of corticosteroid, it decreased by $11 \%$ from baseline $(P<0.01)$. Body weight remained low during the first months of corticosteroid withdrawal, and the sheep received weekly injections of $2 \mathrm{~mL}$ Dexafort for three consecutive weeks to improve their appetite and general wellbeing. By the end of the study, body weight was not different from the start.

3.2. Bone Mineral Density. BMD of the lumbar spine in the osteoporotic sheep decreased over 30\% from baseline after five and half months $(P<0.001$, Figure 3$)$. With cessation 


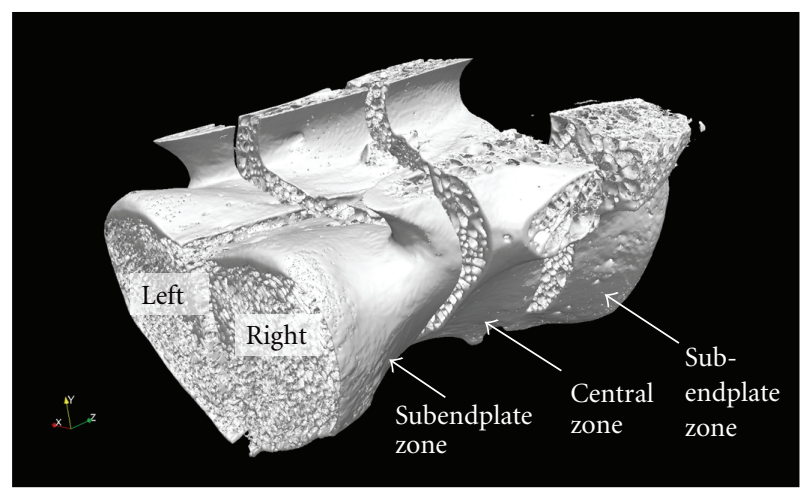

Figure 1: The three dimensions of a sheep vertebral body demonstrating the regions of interest examined (two subendplate zones, central zone, left and right sides).

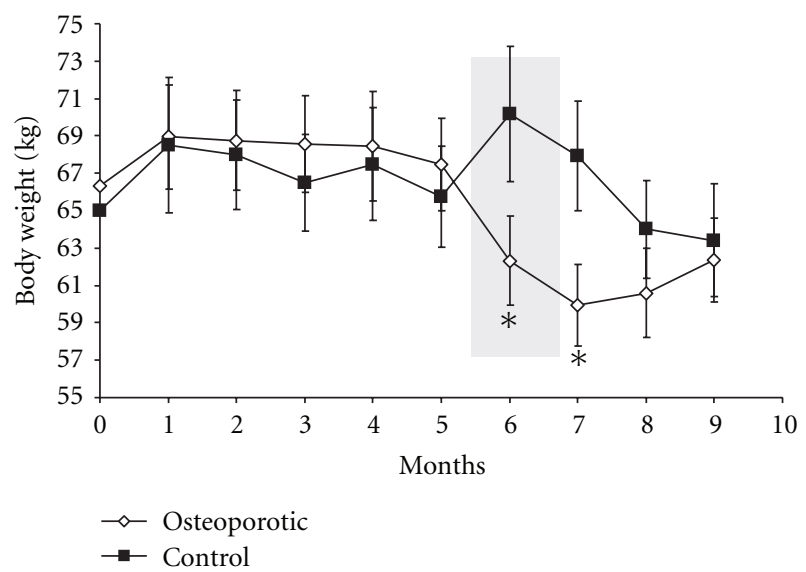

FIGURE 2: Body weight of control and osteoporotic sheep throughout the study. The highlighted gray area shows the period of corticosteroid withdrawal. Body weight was reduced during and one month after withdrawal of corticosteroids $\left({ }^{*} P<0.05\right)$.

of corticosteroid treatment lumbar BMD did not change significantly until the end of the study, at which time BMD of the osteoporotic group was 23\% lower than their baseline and 25\% lower than the control animals $(P<0.001)$.

3.3. Bone Histomorphometry. As the trabecular bone morphometric parameters in both osteoporotic and control groups did not show any difference between the left and right side of each zone, these data were pooled for subsequent analyses. BV/TV in the central zone of the vertebrae in both the control and the osteoporotic sheep was lower than in the endplate zone $(P<0.05$, Figure $4(a))$. Relative to the central zone the sub-endplate zone had higher Tb.Sp, Tb.N and BS/TV in both groups $(P<0.05$, Figures $4(\mathrm{~b}), 4(\mathrm{c})$, and $4(\mathrm{~d})$ ). There was no significant difference in Tb.Th between the central and sub-endplate zones for either group (Figure 4(e)).

Three-months after cessation of corticosteroid administration, the osteoporotic sheep had 23\% less BV/TV in both the central and endplate zone than the controls (Figure 4(a),

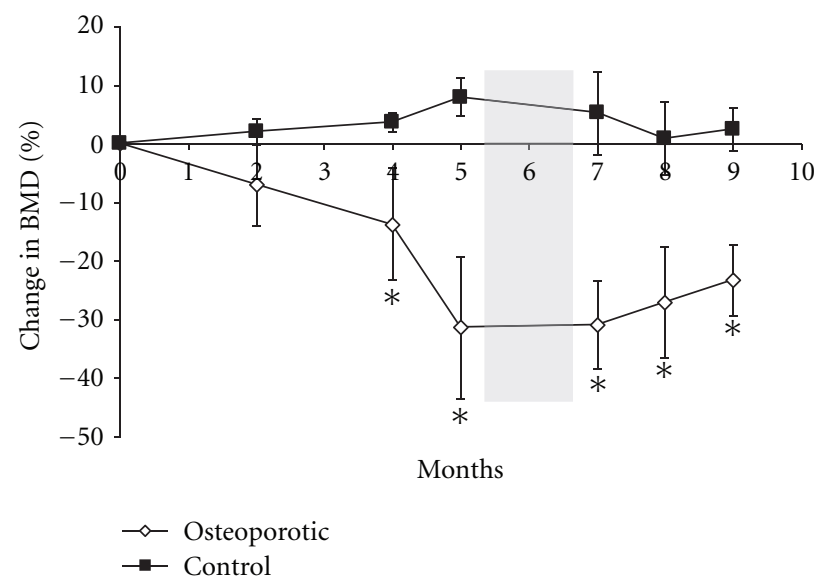

Figure 3: Percentage change in BMD in control and osteoporotic sheep. The highlighted gray area shows the period of corticosteroid withdrawal. BMD of osteoporotic sheep remained lower than controls after withdrawal of corticosteroids $\left({ }^{*} P<0.05\right)$.

$P<0.01)$. Tb.Sp and Tb.N and the bone surface density (BS/TV) in the osteoporotic sheep were not significantly different from the control sheep (Figures 4(b), 4(c), and $4(\mathrm{~d})$ ) although $\mathrm{Tb}$.Th in the osteoporotic sheep was $23 \%$ and $27 \%$ less than the control sheep for the sub-endplate and central zones, respectively, $(P<0.05$, Figure $4(\mathrm{e}))$.

\section{Discussion}

In support of our working hypothesis, this study showed, for the first time, persistence of the osteoporotic condition in the lumbar spine of this animal model after cessation of corticosteroid administration at least for three-months. Further, the vertebral bone of sheep has been shown to be arranged in a heterogeneous manner, with two distinct morphological zones (sub-endplate and central zones), each with very specific trabecular bone density and microstructure, similar to humans.

It is known that rapid withdrawal of corticosteroid can result in acute adrenal insufficiency, which could lead to severe health complications [16]. In our study, the gradual withdrawal of corticosteroids over one month was intended to minimise potential health complications and provides an opportunity for the animals to resume endogenous cortisol production. However, based on our findings and those of other research groups $[18,19]$, it may be necessary to further prolong the withdrawal of steroid (perhaps to seven weeks) to minimise such complications.

On average, BMD of the spine of osteoporotic sheep, 3 months after the cessation of corticosteroid administration, was $25 \%$ lower than the control group, confirming that they maintained their osteoporotic condition. The three-month endpoint was selected to include an important phase of the bone healing process. This confirms the findings from a similar study that demonstrated BMD, microarchitecture, and mechanical competence in the tibia and femur (but not the spine) remained osteopenic six months after the 


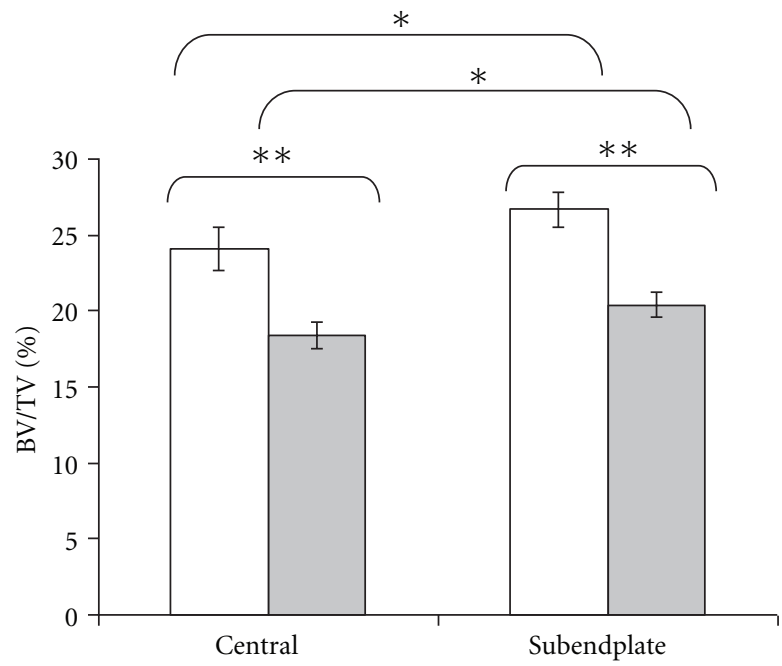

(a)

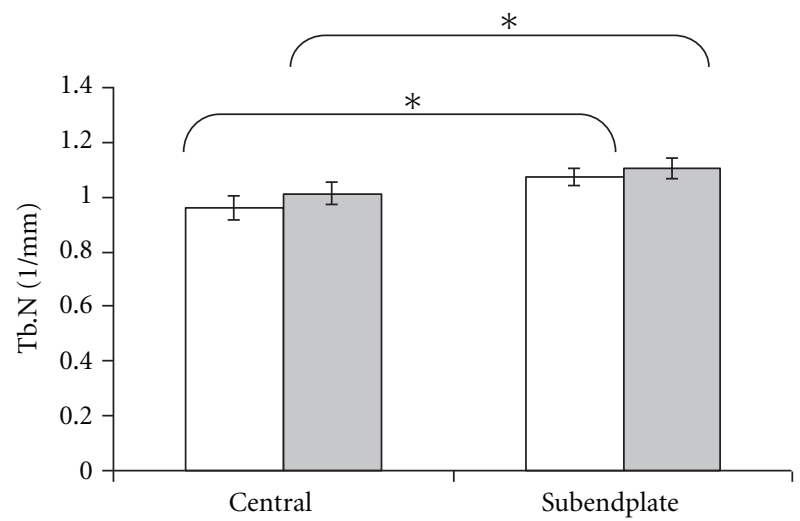

(c)

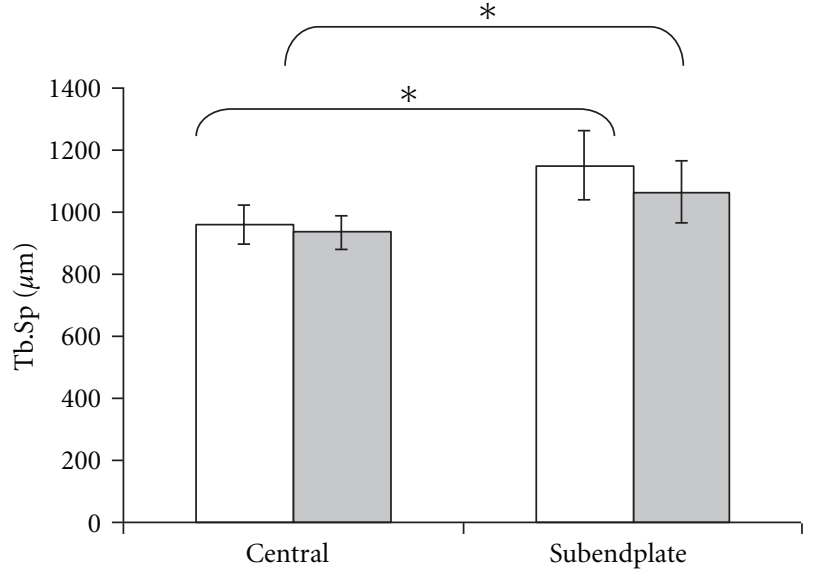

(b)

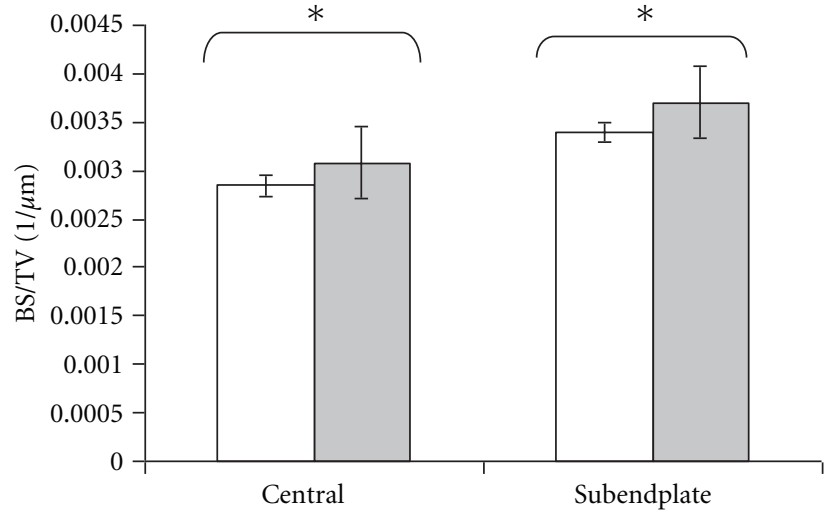

(d)

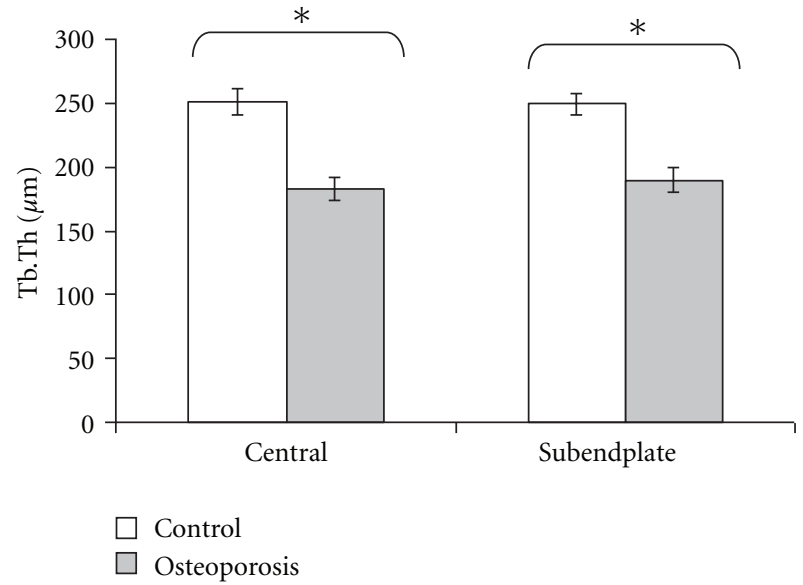

(e)

Figure 4: Comparison of histomorphometric indices of trabecular bone from different regions of lumbar vertebrae in control and osteoporotic sheep $\left({ }^{*} P<0.05,{ }^{* *} P<0.01\right)$.

cessation of glucocorticoid treatment [18]. Further supporting our data, it also showed that BMD in the distal radius of osteoporotic sheep remained significantly lower almost seven months after the end of steroid administration [19].
However, these findings contrasted with more recent work that showed BMD of the spine in an osteopenic sheep model improved to the control level group three-months after the cessation of corticosteroid [20]. This contradictory finding 
was likely due to the presence of normal sex hormone levels in this model compared to our ovariectomised sheep model. These findings demonstrate the significance of synergistic effects of ovariectomy and corticosteroid treatment on bone in the animal model described herein.

An appreciation of bone architecture is essential for the assessment of fracture risk and interpretation of clinical data $[12,21]$. It has been documented that human vertebral bodies are heterogeneous, with two distinct morphological zones (subendplate and central zones), each with very specific trabecular bone density and microstructure [12]. The clinical significance of this heterogeneity has been discussed [12], specifically in relation to the pathogenesis of fractures [22, 23]. Micro-CT analysis shows that the central zone of sheep vertebrae has lower bone volume but thicker trabeculae that are spaced further apart, similar to humans $[12,22]$, although with no lateral variability [24]. The higher bone volume in the sub-endplate region is due mainly to an increase in the number of trabeculae with an associated increased trabecular surface density. The osteoporotic sheep had lower bone volume, mainly as a result of thinner trabeculae. Thus, it is concluded that the reduction of BMD in vertebrae is due mainly to trabecular thinning that leads to biomechanically weaker vertebrae [8]. Such information would have significant clinical application for testing or validation of surgical implants in this animal model. It should be noted, however, that structural components other than trabecular bone, including the endplate, the cortical shell, the posterior elements, osteophytes, and intervertebral discs will also contribute to vertebral strength [21, 25-27]. Thus further characterisation of the structural components of the spine in this animal model is required to extend its applicability for preclinical studies.

In conclusion, the sheep model reported here, which has been validated previously [8-10] is one of severe bone loss that is achieved in a relatively short period, and importantly, in a relatively long-lived, large animal with vertebral trabecular bone microarchitecture similar to humans. The data also demonstrated that bone mass and BMD of this model remained at the osteoporotic level at the end of three-months, indicating that the model is suitable for investigating the early effects of surgical or pharmacological treatments on bone. We acknowledge this osteoporotic sheep model, like all other animal models does not fully mimic either post-menopausal or corticosteroid-induced osteopenia in humans, and the effects of glucocorticoids as well as estrogen deficiency and calcium deficiency are not easily separable. Nonetheless, the aetiology of human postmenopausal osteoporosis is also complex and is not due simply to the loss of oestrogen but rather is influenced by myriad factors including genetic predisposition and body weight, as well as environmental factors, such as vitamin $\mathrm{D}$ and calcium insufficiency, level of exercise or mobility, and various forms of medication, including anticoagulants and glucocorticoids. We believe this animal model is suitable for conducting preclinical trials of novel pharmacologic or surgical interventions for osteoporosis of the lumbar spine as well as other clinically relevant sites. However, a longer follow-up study (six months to one year) after discontinuation of corticosteroids, including additional molecular and histomorphometric investigations, would further confirm the applicability of this model to understand the long-term effects of potential interventions for treating osteoporotic bone.

\section{Conflict of Interests}

The authors declare no conflict of interests (including financial, consultant, institutional, and other relationships) that might lead to bias in this paper.

\section{Acknowledgments}

The authors thank Dr. Peter Self from Adelaide Microscopy for assistance with the $\mu \mathrm{CT}$ analysis, Mr. Adnan Mulaibrahimovic and the staff of the Veterinary Services Division, SA Pathology for animal husbandry.

\section{References}

[1] E. Seeman, "Invited Review: pathogenesis of osteoporosis," Journal of Applied Physiology, vol. 95, no. 5, pp. 2142-2151, 2003.

[2] P. McDonnell, P. E. McHugh, and D. O'Mahoney, "Vertebral osteoporosis and trabecular bone quality," Annals of Biomedical Engineering, vol. 35, no. 2, pp. 170-189, 2007.

[3] L. J. Melton, E. A. Chrischilles, C. Cooper, A. W. Lane, and B. L. Riggs, "Perspective: how many women have osteoporosis?" Journal of Bone and Mineral Research, vol. 7, no. 9, pp. 10051010, 1992.

[4] International Osteoporosis Foundation, What is osteoporosis, 2009, http://www.iofbonehealth.org.

[5] Australia O, Stop the next fracture. In. Osteoporosis Australia, 2009.

[6] Food Drug Administration, "Guidelines for preclinical and clinical evaluation of agents used in the prevention or treatment of postmenopausal osteoporosis," in FDA Division of Metabolism and Endocrine Drug Products, Food and Drug Administration, Washington, DC, USA, 1994.

[7] M. Li, Y. Shen, and T. J. Wronski, "Time course of femoral neck osteopenia in ovariectomized rats," Bone, vol. 20, no. 1, pp. 55-61, 1997.

[8] M. R. Zarrinkalam, H. Beard, C. G. Schultz, and R. J. Moore, "Validation of the sheep as a large animal model for the study of vertebral osteoporosis," European Spine Journal, vol. 18, no. 2, pp. 244-253, 2009.

[9] E. Veigel, R. J. Moore, M. R. Zarrinkalam et al., "Osteopenia in the maxillofacial area: a study in sheep," Osteoporosis International, vol. 22, no. 4, pp. 1115-1121, 2011.

[10] M. R. Zarrinkalam, A. Mulaibrahimovic, G. J. Atkins, and R. J. Moore, "Changes in osteocyte density correspond with changes in osteoblast and osteoclast activity in an osteoporotic sheep model," Osteoporosis International, vol. 23, pp. 13291336, 2011.

[11] J. S. Thomsen, E. N. Ebbesen, and L. Mosekilde, "Static histomorphometry of human iliac crest and vertebral trabecular bone: a comparative study," Bone, vol. 30, no. 1, pp. 267-274, 2002. 
[12] J. S. Thomsen, E. N. Ebbesen, and L. Mosekilde, "Zonedependent changes in human vertebral trabecular bone: clinical implications," Bone, vol. 30, no. 5, pp. 664-669, 2002.

[13] A. M. Parfitt, C. H. E. Mathews, and A. B. Villanueva, "Relationships between surface, volume, and thickness of iliac trabecular bone in aging and in osteoporosis. Implications for the microanatomic and cellular mechanisms of bone loss," The Journal of Clinical Investigation, vol. 72, no. 4, pp. 1396-1409, 1983.

[14] T. S. Keller, T. H. Hansson, A. C. Abram, D. M. Spengler, and M. M. Panjabi, "Regional variations in the compressive properties of lumbar vertebral trabeculae. Effects of disc degeneration," Spine, vol. 14, no. 9, pp. 1012-1019, 1989.

[15] X. E. Guo and C. H. Kim, "Mechanical consequence of trabecular bone loss and its treatment: a three-dimensional model simulation," Bone, vol. 30, no. 2, pp. 404-411, 2002.

[16] R. Bouillon, "Acute Adrenal Insufficiency," Endocrinology and Metabolism Clinics of North America, vol. 35, no. 4, pp. 767775, 2006.

[17] A. M. Parfitt, M. K. Drezner, F. H. Glorieux et al., "Bone histomorphometry: standardization of nomenclature, symbols, and units. Report of the ASBMR Histomorphometry Nomenclature Committee," Journal of Bone and Mineral Research, vol. 2, no. 6, pp. 595-610, 1987.

[18] S. Schorlemmer, C. Gohl, S. Iwabu, A. Ignatius, L. Claes, and P. Augat, "Bone histomorphometry: standardization of nomenclature, symbols, and units. Report of the ASBMR Histomorphometry Nomenclature Committee," Journal of Bone and Mineral Research, vol. 18, no. 11, pp. 2010-2015, 2003.

[19] J. Goldhahn, A. Jenet, E. Schneider, and C. A. Lill, "Slow rebound of cancellous bone after mainly steroid-induced osteoporosis in ovariectomized sheep," Journal of Orthopaedic Trauma, vol. 19, no. 1, pp. 23-28, 2005.

[20] M. Ding, L. Cheng, P. Bollen, P. Schwarz, and S. Overgaard, "Glucocorticoid induced osteopenia in cancellous bone of sheep: validation of large animal model for spine fusion and biomaterial research," Spine, vol. 35, no. 4, pp. 363-370, 2010.

[21] P. A. Hulme, S. K. Boyd, and S. J. Ferguson, "Regional variation in vertebral bone morphology and its contribution to vertebral fracture strength," Bone, vol. 41, no. 6, pp. 946-957, 2007.

[22] H. Gong, M. Zhang, L. Qin, K. K. H. Lee, X. Guo, and S. Q. Shi, "Regional variations in microstructural properties of vertebral trabeculae with structural groups," Spine, vol. 31, no. 1, pp. 24-32, 2006.

[23] M. D. Antonacci, D. S. Hanson, A. Leblanc, and M. H. Heggeness, "Regional variation in vertebral bone density and trabecular architecture are influenced by osteoarthritic change and osteoporosis," Spine, vol. 22, no. 20, pp. 2393-2402, 1997.

[24] X. Banse, J. P. Devogelaer, E. Munting, C. Delloye, O. Cornu, and M. Grynpas, "Inhomogeneity of human vertebral cancellous bone: systematic density and structure patterns inside the vertebral body," Bone, vol. 28, no. 5, pp. 563-571, 2001.

[25] L. D. Hordon, M. Raisi, J. E. Aaron, S. K. Paxton, M. Beneton, and J. A. Kanis, "Trabecular architecture in women and men of similar bone mass with and without vertebral fracture: $i$. two-dimensional histology," Bone, vol. 27, no. 2, pp. 271-276, 2000.

[26] E. K. Simpson, I. H. Parkinson, B. Manthey, and N. L. Fazzalari, "Intervertebral disc disorganization is related to trabecular bone architecture in the lumbar spine," Journal of Bone and Mineral Research, vol. 16, no. 4, pp. 681-687, 2001.
[27] L. Mosekilde, "Age-related changes in bone mass, structure, and strength-effects of loading," Zeitschrift für Rheumatologie, vol. 59, no. 1, pp. 1-9, 2000. 


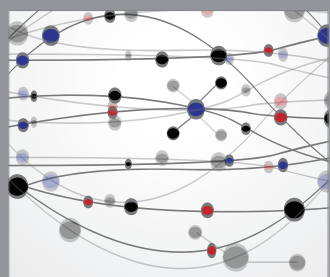

The Scientific World Journal
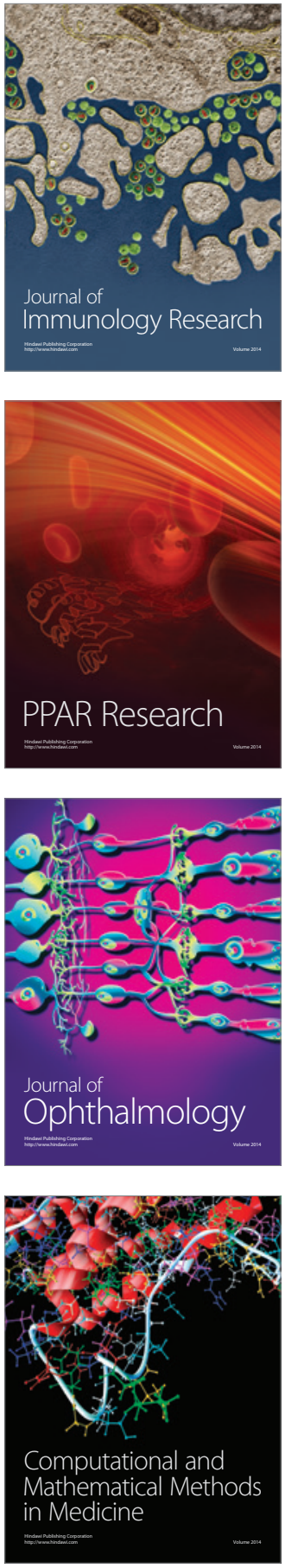

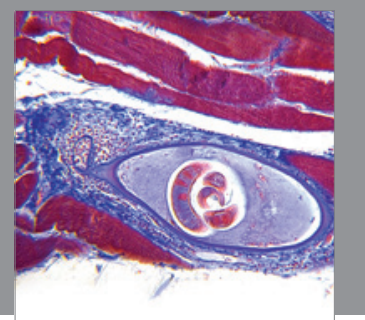

Gastroenterology

Research and Practice
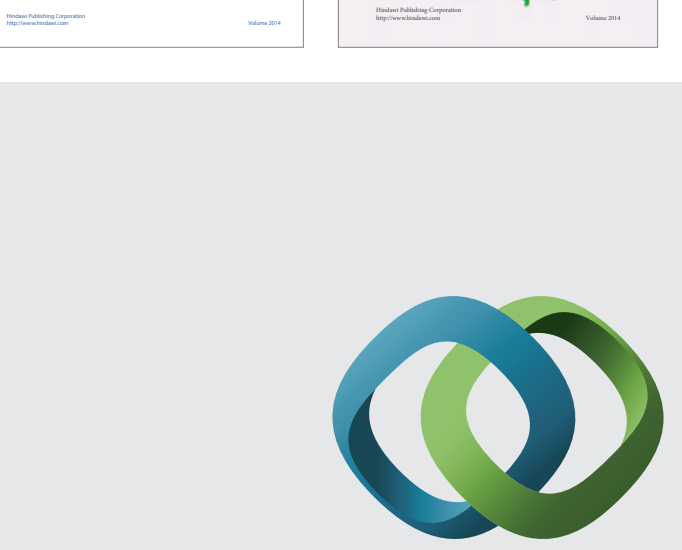

\section{Hindawi}

Submit your manuscripts at

http://www.hindawi.com
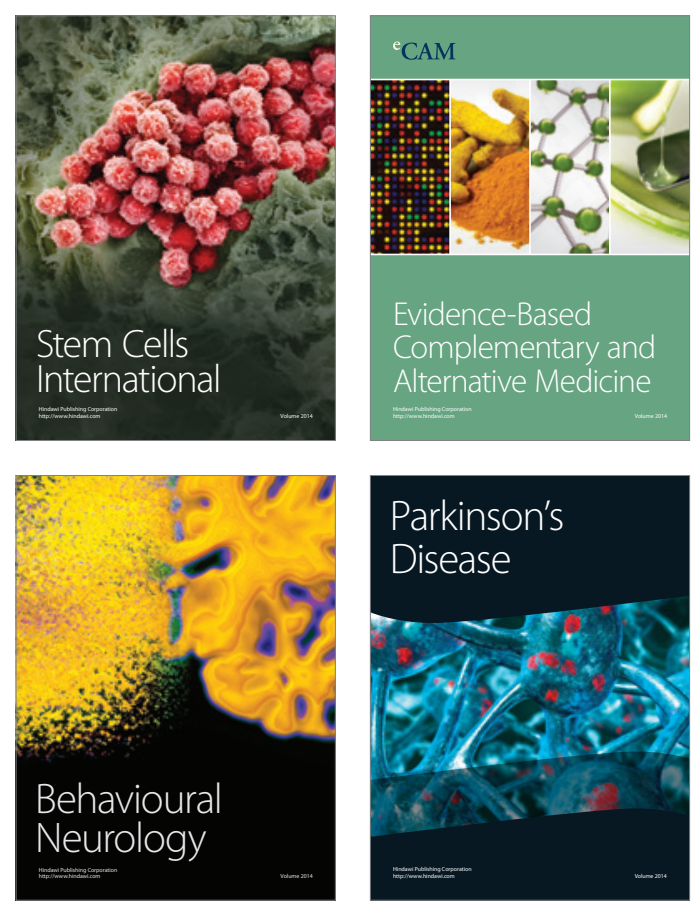

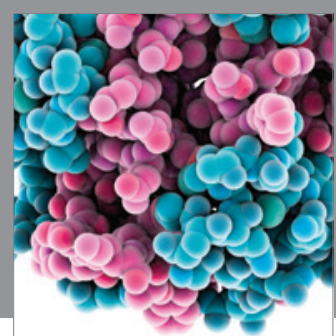

Journal of
Diabetes Research

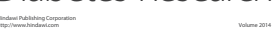

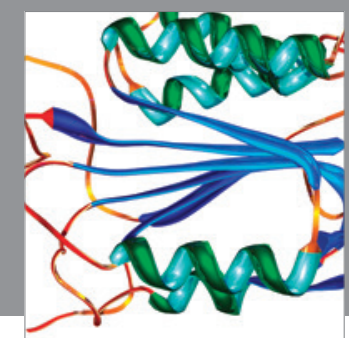

Disease Markers
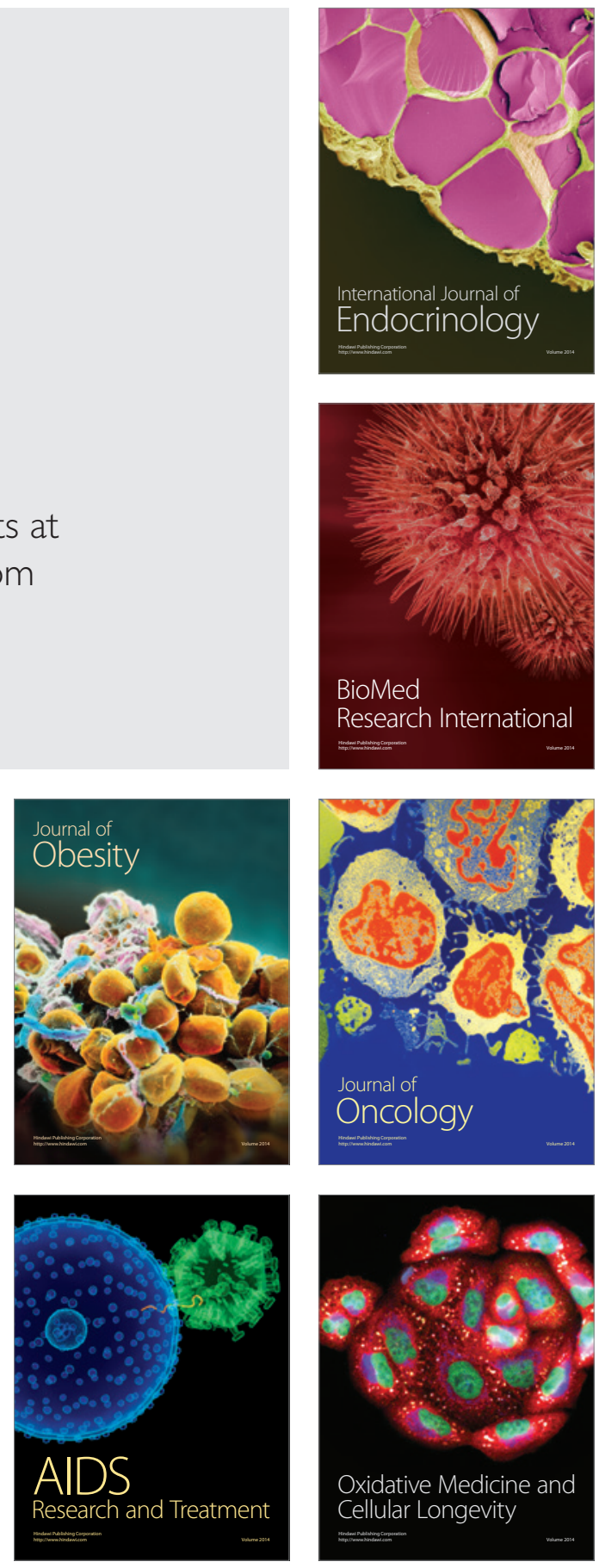